Théologiques

Théologiques

\title{
Le monachisme chrétien orthodoxe vu au prisme des relations
} Église-État Survol à l'occasion d'un colloque dédié à l'ancien Porphyre

\section{Athanase Giocas}

Volume 23, numéro 1, 2015

URI : https://id.erudit.org/iderudit/1040870ar

DOI : https://doi.org/10.7202/1040870ar

Aller au sommaire du numéro

Éditeur(s)

Faculté de théologie et de sciences des religions, Université de Montréal

ISSN

1188-7109 (imprimé)

1492-1413 (numérique)

Découvrir la revue

Citer cet article

Giocas, A. (2015). Le monachisme chrétien orthodoxe vu au prisme des relations Église-État : survol à l'occasion d'un colloque dédié à l'ancien Porphyre. Théologiques, 23(1), 167-180. https://doi.org/10.7202/1040870ar

\section{Résumé de l'article}

Pour mettre en oeuvre un modèle ecclésiologique qui corresponde à l'enseignement de l'ancien Porphyre, la relation intime entre histoire et théologie doit être plus profondément appréciée. Dans cet esprit, l'article expose les grandes lignes d'une réflexion plus large sur le parcours historique du monachisme orthodoxe, se référant à la conception traditionnelle du développement du monachisme au $\mathrm{IV}^{\mathrm{e}}$ siècle, ainsi qu'à certaines expressions plus contemporaines du monachisme. Dans tous les cas, le monachisme agit comme une sorte de contrepoids à la présence continue de forces sécularisantes qui agissent sur la réalité ecclésiale de l’Église et sa mission. 


\title{
Le monachisme chrétien orthodoxe vu au prisme des relations Église-État
} Survol à l'occasion d'un colloque dédié à l'ancien Porphyre ${ }^{1}$

\author{
Athanase Giocas* \\ Théologie \\ Institut de théologie orthodoxe de Montréal (Canada)
}

\section{Introduction}

Le $12^{\mathrm{e}}$ Colloque de théologie orthodoxe de l'Université de Sherbrooke était consacré à l'ancien Porphyre (1906-1991). Ce rassemblement stimulant fut l'occasion de prendre connaissance de la vie et de l'œuvre de Porphyre, ainsi que de la tradition plus générale des anciens dans la spiritualité orthodoxe ${ }^{2}$.

1. Cet article est une version révisée d'une conférence présentée à Montréal le 21 avril 2012 lors du $12^{\mathrm{e}}$ Colloque de théologie orthodoxe organisé par l'Institut de théologie orthodoxe de Montréal, affilié à l'époque avec la Faculté de théologie et d'études religieuses de l'Université de Sherbrooke, et associé depuis 2015 avec la Faculté de théologie et de sciences religieuses de l'Université Laval.

* Athanase Giocas est docteur en droit de l'Université de Montréal. Il possède une triple formation en génie (McGill), droit (McGill, Francfort) et théologie (Sherbrooke). Il occupe des postes de direction au sein de la Fondation hellénique de bourses d'études et de l'Institut de théologie orthodoxe de Montréal. Ses champs de recherche incluent le droit et la religion, la théologie orthodoxe et la philosophie juridique. Il a récemment publié (2016) Le bien justifié. Une lecture contemporaine de la synthèse philosophico-juridique de Vladimir S. Soloviev, Presses de l'Université Laval (Diké). L'auteur désire remercier les deux évaluateurs anonymes de ce texte pour leurs suggestions et commentaires.

2. Dans le christianisme orthodoxe, le titre d' "ancien » est réservé aux pères spirituels les plus charismatiques issus du milieu monastique. Dans la tradition grecque, un ancien est souvent appelé "géron", provenant du mot grec « $\gamma \varepsilon ́ \rho \circ v \tau \alpha$ » qui désigne littéralement un «vieillard». Tandis que dans la tradition russo-slave, le mot «starets » (translitéré du russe «ста́рец», qui lui aussi désigne un «vieillard») est utilisé. Pour une introduction en français à la tradition spirituelle de l'Église orthodoxe, nous recommandons les études de Deseille (2003) et Behr-Sigel (1989). 
En somme, l'ancien Porphyre est largement reconnu comme l'exemple d'un homme qui, à travers l'ascèse, s'est donné au service de Dieu et de tous ceux qui ont reçu de lui un bon conseil. Sans aucun doute, Porphyre a été une bénédiction pour l'Église, et encore plus pour les milliers de pèlerins qui l'ont connu personnellement et qui ont pu voir leur foi renforcée par sa clairvoyance et son discernement. L'œuvre notamment pastorale de l'ancien Porphyre dévoila la manifestation possible de la présence de Dieu parmi nous, une présence que la sagesse porphyrienne rendait perceptible avant tout dans l'amour et la paix. D'après divers témoignages, les conseils de Porphyre renvoyaient sans cesse aux paroles du Christ, à l'amour céleste du Christ et au pouvoir transformateur de la grâce divine.

\section{Parcours argumentatif}

Le titre de cette courte réflexion - «Le monachisme chrétien orthodoxe vu au prisme des relations Église-État» — peut naturellement paraître un peu étrange au sein d'un colloque sur l'ancien Porphyre. Toutefois, nous prétendons que la sagesse porphyrienne demeure bel et bien au cœur du sujet. Notre but est donc de présenter les grandes lignes d'une réflexion sur les enjeux contemporains de la vie chrétienne, en situant notamment le monachisme orthodoxe dans une trajectoire historique beaucoup plus large que d'habitude. Nous espérons que cela pourra élargir la portée du regard critique collectif qui s'impose pour évaluer les différentes visions concurrentes de la paroisse orthodoxe en Amérique du Nord au Xxi siècle $^{3}$. Nous réalisons que le voyage que nous proposons à travers l'espace et le temps peut, à certains égards, paraître trop sommaire. Une présentation plus étoffée d'un ou même plusieurs éléments constitutifs de notre enchâ̂nement argumentatif comporterait sans doute son propre intérêt, mais risquerait également de priver l'argument global de sa visée et de son effectivité en tant que point de démarrage accessible d'un sujet vaste et fort complexe. Ce qui suit n'aspire aucunement à être plus qu'une utile première approche d'une problématique interdisciplinaire essentielle mais peu étudiée.

Avec cette réserve clairement énoncée, nous pouvons entamer notre réflexion, qui se déploie en deux parties. Premièrement, nous mettons au point la compréhension classique de la relation causale entre le développe-

3. Précisons que l'expérience de l'«Amérique du Nord» à laquelle nous faisons référence se limite à toutes fins pratiques aux cas du Canada et des États-Unis. 
ment du monachisme d'un côté et l'établissement du christianisme comme religion impériale de l'autre. Ici, une claire allusion est faite au récit dont nous sommes tous familiers et selon lequel le développement du monachisme au $\mathrm{IV}^{\mathrm{e}}$ siècle découle de la fin de la période des persécutions de l'Église des catacombes et du début de l'implantation graduelle du christianisme comme religion officielle dans l'Empire romain. À partir de cette compréhension traditionnelle peut se dégager le fondement d'un modèle théorique pour analyser en contexte le monachisme au sein d'un environnement politico-religieux quelconque.

Dans la deuxième partie de notre réflexion, nous appliquons ce modèle à deux phénomènes contemporains afin d'illustrer le genre d'analyse fructueuse à laquelle il peut donner lieu. Le premier phénomène consiste en la vie de l'ancien Porphyre et s'étend au contexte plus large du monachisme dans la Grèce moderne. Le deuxième phénomène concerne le développement monastique au sein de l'Église grecque en Amérique du Nord. Le fait de bondir $\mathrm{du} \mathrm{IV}^{\mathrm{e}}$ au $\mathrm{XX}^{\mathrm{e}}$ siècle dans un si court espace est certes une démarche assez peu conventionnelle, mais si elle est employée ici, c'est à la seule fin de défricher quelques pistes de pensée innovatrices et utiles au sujet de la tradition vivante de l'orthodoxie.

\section{Les contours du récit traditionnel}

Le récit traditionnel est propagé dans tous les ouvrages sur le christianisme. Tel qu'il y est présenté, l'épanouissement général du monachisme au $\mathrm{IV}^{\mathrm{e}}$ siècle est considéré comme résultant de la fin de la période des persécutions et du début de l'appropriation du christianisme par l'Empire romain. Ce processus est souvent exprimé comme le triomphe du christianisme. Comme le décrit Festugière (1961, 18-19) dans le premier volume de son étude sur Les Moines d'Orient:

L'Empire est officiellement chrétien, le paganisme n'est plus que toléré, à la fin du $\mathrm{IV}^{\mathrm{e}}$ siècle il ne l'est même plus, et a fortiori au V $\mathrm{V}^{\mathrm{e}}$. Si l'Empereur est officiellement chrétien, il n'y a plus désavantage, mais avantage, à être chrétien: et donc charges, titres, pouvoirs et honneurs, sans être uniquement réservés aux chrétiens, du moins au $\mathrm{IV}^{\mathrm{e}}$ siècle, s'amoncellent désormais sur les chrétiens. Il y a une cour chrétienne à Constantinople, avec une infinité de dignitaires et de flatteurs. Dès lors, pour le chrétien sérieux, qui prend à la lettre les vérités de la foi, le problème se pose: peut-il encore, dans le monde, suivre Jésus, porter la croix de Jésus? Peut-il encore, dans le monde, être sauvé? Beaucoup résoudront ce problème par la négative et s'enfuiront au désert. 
Membre de la diaspora russe, le père Alexandre Schmemann (19211983) s'installa aux États-Unis en 1951 et fut un des plus importants auteurs orthodoxes du $\mathrm{xx}^{\mathrm{e}}$ siècle. Selon lui, la vie de saint Antoine, telle que rédigée par saint Athanase, représente le modèle initial de l'idéal monastique. Pour sa part, Athanase décrit la solitude pieuse des ermitages d'Égypte comme suit:

Dans les montagnes, les ermitages étaient donc comme des tentes remplies de chœurs divins, chantant des psaumes, lisant les Écritures, jeûnant, priant, se réjouissant dans l'espérance des biens futurs, travaillant pour faire l'aumône et menant une vie d'amour mutuel et de concorde. On pouvait vraiment y voir comme une région à part de piété et de justice. Il n'y avait là personne qui souffrît l'injustice, $[. .$.$] mais une multitude d'ascètes, avec pour$ unique préoccupation la vertu. (Vie d'Antoine, 255)

Un autre personnage illustre de la diaspora russe, le père Georges Florovsky (1893-1979). Il nous renvoie au langage révolutionnaire pour tisser le lien entre l'Église des martyrs et le monachisme sous l'Empire chrétien. Florovsky soutient précisément que l'Église était un type de mouvement de résistance sous l'Empire païen et que le monachisme devient désormais un mouvement de résistance de forme plus permanente au sein de la société chrétienne. L'épanouissement du monachisme au IV ${ }^{\mathrm{e}}$ siècle est donc une tentative d'échapper aux compromis nécessairement liés au pouvoir impérial et de bâtir une société chrétienne autonome à l'extérieur des limites de l'Empire (Florovsky 1957, 138). Il est superflu de s'attarder davantage sur un récit tellement répandu de l'histoire des origines du développement monastique au IV siècle. Retour donc vers le futur et à l'ancien Porphyre.

\section{Le cas de l'ancien Porphyre}

L'ancien Porphyre est certainement issu du monachisme orthodoxe. Il a souvent parlé du monachisme et de son importance au sein de l'Église. Porphyre déclara même que l'Église a pu être préservée historiquement en raison du monachisme (Porphyrios 2005, 171). Pour mieux illustrer cette idée de préservation, on pourrait penser à la tradition de transcription des textes religieux de la part des scribes monastiques, une activité essentielle pour la transmission des connaissances. On pourrait également penser au développement des offices religieux au sein des monastères. Le canon liturgique qui est toujours utilisé dans l'Église orthodoxe trouve en fait son origine dans les monastères et surtout dans le Typikon du monastère de Saint-Sava à Jérusalem (Getcha 2009, 52). 
Toutefois, l'explication que donne l'ancien Porphyre sur cette question relève d'un ordre très différent, qui dépasse de beaucoup les contributions techniques ou facilement saisissables du monachisme. À cet égard, Porphyre souligne surtout la contribution mystique de la prière du moine, une prière dont les réverbérations atteignent le monde entier, et même ceux qui se trouvent très loin. En accord avec son enseignement plus général, les écrits de Porphyre sur le monachisme sont axés sur l'amour et l'humilité. Pour Porphyre, le moine se retire au monastère, non pas pour se distancer des autres, mais avec le désir que tous soient sauvés (Porphyrios 2005, 89). Porphyre disait que "[n]i le travail, ni les prosternations, ni les signes de croix n'attirent la grâce de Dieu... Il faut aller au-delà des aspects formels. Tout ce qui est fait doit être fait avec amour» (Porphyrios 2005, 95, nous traduisons). L'extrait suivant est révélateur:

Si un moine veut progresser dans un monastère, il doit s'engager volontairement dans la lutte spirituelle sans la pression de quelqu'un d'autre. Il a besoin de tout faire avec joie et empressement, et non pas comme une corvée. Un moine n'est pas une personne qui est obligée de faire quelque chose mécaniquement et à contrecœur. Quoi qu'il fasse, il le fait uniquement par amour pour l'époux céleste, par amour (éros) divin. Le monachisme ne doit pas être une fuite négative du monde, mais une fuite par amour divin et vénération divine. (Porphyrios 2005, 158, nous traduisons)

Pourtant, la vie de Porphyre nous expose à des aspects du monachisme non-traditionnels, dont le plus important est qu'il s'est lui-même, pour des raisons de santé, retiré de la Sainte Montagne, le mont Athos, pour servir l'Église dans le monde. Pour une grande partie de sa vie, il s'occupa ainsi de la chapelle d'un hôpital en plein centre d'Athènes. Porphyre est un exemple fort de quelqu'un qui a vécu la foi au-delà des formes traditionnelles. Il était un moine dans le monde, et plus précisément dans le monde de la Grèce moderne du $\mathrm{xx}^{\mathrm{e}}$ siècle. À l'époque, tout comme à l'heure actuelle, l'Église orthodoxe grecque jouait un rôle des plus importants dans la sphère publique en Grèce (voir Papastathis 2001). La Constitution de la Grèce reconnaît formellement la primauté de la foi orthodoxe. Des arrangements législatifs très compliqués régissent les relations entre l'État et l’Église, selon l'idéal de la symphonie entre le pouvoir temporel et le pouvoir spirituel. Mais, à la limite, ce chevauchement constitutionnel entre un régime politique démocratique et la reconstitution de certains aspects de la théocratie byzantine suscite plusieurs interrogations. 
Du point de vue pratique, la mission de l'Église est souvent encombrée. Par exemple, on sait que Porphyre travailla physiquement (en agriculture et en tricot) pour gagner sa vie, malgré les divers privilèges financiers de l'Église. Lorsqu'il fonda un tout petit monastère dans sa retraite, lui aussi fut victime de la bureaucratie lente et inefficace de l'État grec. Sur un plan plus théorique, selon le modèle fondé sur le récit classique de l'épanouissement du monachisme, la forte présence du monachisme en Grèce peut s'expliquer clairement par le fait que l'Église continue d'être formellement liée au pouvoir temporel de l'État. Dans ce sens, le récit traditionnel trouve toute son incidence dans le contexte politico-religieux de la Grèce moderne.

La relation entre l'Église et l'État en Grèce suscite quand même de vives discussions. Par exemple, Christos Yannaras, dans certains écrits datant des années 70 , critique très nettement la situation du régime des relations entre l'Église et l'État en Grèce et dénonce la tentation de transformer la réalité ecclésiologique et œcuménique de l'Église en simple institution étatique. Plus précisément, Yannaras $(1983,201)$ put même affirmer en 1975, dans un grand journal d'Athènes, que la séparation entre l'Église et l'État en Grèce serait en fin de compte incontournable. Plus récemment, c'est le Métropolite grec Nicolas Hadjinicolaou qui plaide ouvertement pour la séparation entre l'Église et l'État en Grèce. Son raisonnement est assez subtil. Pour le Métropolite Nicolas, cette séparation s'est déjà réalisée du point de vue spirituel. Dans ses interventions publiques, il parle même d'un système étatique athée, face auquel l'Église doit recouvrer son indépendance pour mettre fin aux forces sécularisantes qui agissent sur elle (Theocharis 2011). Cette opinion est loin d'être partagée par la majorité de la hiérarchie de l'Église grecque.

Toutefois, et surtout en tenant compte de la crise actuelle en Grèce, il serait de plus en plus difficile de nier le lien entre le statut constitutionnel de l'Église en Grèce et la légitimation récurrente d'une classe politique des plus abîmées au niveau de l'éthique. C'est ce qui, à notre avis, fait la pertinence du point que soulève le Métropolite Nicolas avec beaucoup de discernement: l'État a tiré plusieurs bénéfices du régime actuel, non seulement sur le dos de l'Église, mais en défavorisant profondément à la fois la mission de l'Église et celle de l'État.

\section{Le monachisme au sein de l'Église grecque en Amérique du Nord}

En Amérique du Nord, la séparation entre l'Église et l'État est bien établie. De toutes façons, les orthodoxes représentent un très petit pourcentage de 
la population ${ }^{4}$. Socialement et politiquement, la réalité de l'Amérique du Nord est complètement différente de celle de la Grèce moderne. Par conséquent, on ne s'attendrait guère à voir l'impulsion du développement du monachisme orthodoxe se conformer au récit traditionnel. Toutefois, au cours des vingt dernières années, on a pu assister à une floraison du monachisme au sein de l'Église grecque en Amérique du Nord dont la rapidité et l'étendue ont quelque chose de miraculeux. Nous faisons surtout référence à la fraternité du confesseur Éphrem et aux quelque vingt monastères qu'il a fondés aux États-Unis et au Canada. L'ancien Éphrem, un moineconfesseur au monastère de Philotheou au mont Athos, s'est implanté aux États-Unis vers les années 1980-90. Et l'épanouissement monastique auquel il présida n'est pas allé sans certaines controverses. Très souvent, ces controverses se rapportent à la promotion d'un sectarisme interorthodoxe de la part des disciples d'Éphrem, surtout à l'encontre des membres des communautés et paroisses existantes. En plus des monastiques sous son contrôle immédiat, l'influence d'Éphrem s'étend à un grand nombre de laïcs qui ont développé un fort sentiment d'obéissance envers lui. Nous n'avons pas l'intention de prendre position dans un débat sur l'exactitude de certains reproches contre le mouvement d'Éphrem, d'autant plus que ces reproches sont fondés sur du ouï-dire, c'est à dire des paroles de tiers dont la crédibilité est difficile à évaluer. Cependant, une émission d'une chaîne locale de télévision américaine s'est déjà penchée - entre autres - sur les méthodes de recrutement des moines au sein des monastères d'Éphrem (Fox News KVOA TV 4 2006).

Y avait-il des monastères au sein de l'Église grecque avant l'arrivée d'Éphrem? Oui, mais le cheminement de certains est assez catastrophique.

4. Cela ne signifie pas que les hauts représentants de l'État n'entretiennent aucune relation avec la hiérarchie de l'Église orthodoxe. À titre d'illustration, l'Archevêque Démétrios de l'Archevêché orthodoxe grec de l'Amérique, tout comme ses prédécesseurs, cultive avec succès de solides relations avec plusieurs hautes instances politiques de l'État américain. En plus de son rôle comme représentant par excellence de la communauté influente des Américains d'origine grecque, l'Archevêché promeut plusieurs dossiers, en particulier la reconnaissance de la situation précaire du Patriarcat de Constantinople en Turquie, la gestion des crises grecques de l'économie et des réfugiés, et la construction d'un sanctuaire sur le site du World Trade Center en remplacement de l'église orthodoxe de St Nicolas détruite lors des attentats du 11 septembre 2001. En tout cas, nul ne prétend que l'Église orthodoxe souhaite à travers ces relations publiques reconfigurer l'État américain selon une vision politicoreligieuse quelconque, une idée qui serait anathème, vu l'importance historique de l'enracinement du principe de la séparation entre l'État et la religion aux États-Unis. 
Mentionnons deux exemples. Le monastère de Ste-Irène à Astoria, dans le quartier du Queens de New York, qui a été le centre d'un scandale financier et sexuel. Ce monastère a été fondé en 1972 et faisait, à l'origine, partie de l'Église vieux-calendariste ${ }^{5}$. Le monastère a été reçu dans l'Église orthodoxe canonique en 1998. Les deux moines impliqués dans le scandale (l'abbé Paissios, métropolite, qui a fondé le monastère en 1972 et son assistant Vikentios, évêque) ont été défroqués en mars 2012 par le SaintSynode du Patriarcat de Constantinople. Les diverses enquêtes menées par l’Église n'ont jamais été rendues publiques. Un peu plus près de nous, mentionnons très brièvement ce qui a été considéré comme le premier monastère de l'Église canonique grecque au Québec. Martin Robert Chartray fut d'abord un prêtre catholique; reçu par l'Église orthodoxe, il établit en 1991 un monastère à Stanhope, à $50 \mathrm{~km}$ au sud de la ville de Sherbrooke, près de la frontière avec les États-Unis. Dans des conditions mystérieuses, le père Martin se suicida quelques mois après, donnant une fin assez tragique à ce monastère ${ }^{6}$.

Parvenu à ce point, nous aimerions soulever l'hypothèse selon laquelle le récit traditionnel peut toujours expliquer la situation en Amérique du Nord. Même si les diverses autorités étatiques en Amérique du Nord n'entretiennent aucune relation politique significative avec l'Église orthodoxe, il se peut que l'ombre d'une tendance impériale persiste au sein même de l'Église. Autrement dit, le modèle analytique fondé sur le récit traditionnel envisage une opposition entre le monachisme et les forces séculières. Le fait que ces forces se soient déplacées à travers le temps et l'espace, ou plutôt de l'État-nation à l'Église-nation, peut facilement être intégré à notre modèle. Dans cette perspective, les monastères d'Éphrem en Amérique du Nord ne font que révéler une crise d'identité plus générale au sein de l'Église orthodoxe grecque, crise liée à la sécularisation qui s'opère à l'intérieur même de la réalité ecclésiale, telle que vécue par les croyants. Si cela est vrai, des incidences importantes dans nos façons de vivre la foi en découle, y compris le très grand danger que pose l'enracine-

5. Les Églises vieux-calendaristes grecques sont le résultat d'une série de schismes et divisions à l'intérieur de l'Église orthodoxe en Grèce, à la suite de l'adoption de certaines révisions au calendrier julien en 1924. Ces révisions visaient une meilleure correspondance entre le calendrier orthodoxe et le calendrier grégorien qui est de rigueur dans une grande majorité de pays à travers le monde.

6. Sur l'historique des évènements entourant ce monastère, voir Sahas (2002, 279-388) et Hustak (1992). 
ment d'un monachisme de plus en plus reclus et isolé du monde, en réponse à une vision de plus en plus séculière de la paroisse.

Cette réflexion nous mène à une constatation assez paradoxale au sujet de l’Église de la diaspora grecque. Même si aucune force étatique ne réclame l'Orthodoxie comme religion officielle en Amérique du Nord, il se pourrait qu'une problématique similaire s'y soit implantée. Nous avançons l'hypothèse subsidiaire selon laquelle la cause serait liée à la tendance que possède une certaine hiérarchie, formée en Grèce, d'importer des considérations propres à la réalité de la Grèce moderne - ou à une conception nostalgique et idéalisée de Byzance ou de la période post-byzantine. Dans tous les cas, nous faisons face au risque d'une vie paroissiale de plus en plus déroutante, en raison d'une vision démesurément dogmatique, ainsi que du clivage croissant entre la paroisse et le monastère, qui atteint d'inquiétantes proportions.

\section{Conclusion}

Les orthodoxes se considèrent comme les gardiens d'une tradition chrétienne des plus authentiques, tradition qui reflète le mieux l'esprit de l'Église ancienne. Mais, quelques fois, nous éprouvons de la difficulté à regarder d'un œil critique nos pratiques et nos attitudes. Depuis l'Église ancienne, les rapports entre la conception de la religion publique de l'État, d'une part, et le monachisme, de l'autre, est parfois difficile à discerner. Il nous semble toutefois qu'un fil conducteur ressort assez clairement de l'expérience historique de l'Église orthodoxe sous la forme d'une forte tendance à réagir aux forces sécularisantes qui percutent l'Église historique en se retranchant sur l'intériorité. En plus de l'épanouissement général du monachisme, ce retranchement est aussi bien démontré par la pratique de recruter des évêques exclusivement parmi les rangs des moines, ainsi que par la vie très intense de monastères établis dans la capitale de l'Empire, à Constantinople, à l'époque de Byzance.

Durant la période post-byzantine, une fois que le pouvoir temporel de l'empire chrétien s'est éteint, dans la liturgie comme dans la communauté, l'évêque prend la place de l'autorité impériale et l'Église se recentre plus directement sur la mission pastorale des communautés sous occupation étrangère. Mais depuis l'indépendance de la Grèce, la situation devient un peu confuse, en partie parce que certaines régions, très importantes culturellement, ne se sont jamais libérées. Toutefois, se retrouver théologiquement dans le désarroi historique des pays orthodoxes est absolument 
nécessaire pour mieux saisir la situation contemporaine de l'Église orthodoxe en Amérique du Nord et établir une trajectoire pour l'avenir.

En conclusion, rappelons-nous de l'enseignement de Porphyre sur la vie en Christ:

Géron enseignait que le caractère fondamental de la vie spirituelle en Christ, le grand mystère de notre foi, c'est l'union, le sens de notre identification avec nos frères, le fait de nous soulager les uns les autres de nos fardeaux, de vivre, de faire l'expérience de l'autre comme de nous-mêmes, de dire: "Seigneur Jésus-Christ, aie pitié de moi » et dans ce «moi", de faire nôtre la douleur de l'autre et son problème, de souffrir comme il souffre, de nous réjouir comme il se réjouit: nôtre est sa chute, nôtre est sa rédemption. (Ioannidis 2005, 55)

Porphyre, par son humilité et son amour, nous a légué un testament spirituel très précieux. Ce testament soutient la nature libertaire du christianisme, c'est-à-dire l'importance de promouvoir la rencontre avec le Christ dans le libre-arbitre. Et si l'ancien Porphyre devient la norme idéale d'un ancien, il est aussi la norme au prisme de laquelle les anciens d'aujourd'hui devront être jugés. En cherchant le rétablissement d'une ecclésiologie plus saine et plus authentique, un examen renouvelé du modèle des relations Église-État est d'une portée analytique immense pour mieux saisir le trajet historique de l'Église orthodoxe, pour mieux comprendre la relation intime entre histoire et foi. Mais cette relation ne devrait jamais éclipser l'amour ou la liberté promus par la sagesse porphyrienne sur la base de l'Évangile.

\section{Épilogue}

Depuis l'élaboration initiale, ainsi que la première présentation publique de cette réflexion, plusieurs évènements marquants dans la vie de l'Église orthodoxe ont eu lieu qui sont directement pertinents au principal fil historico-théorique que nous avons essayé de tisser et de mettre en lumière. Tout d'abord, l'ancien Porphyre a été canonisé par le Patriarcat œcuménique de Constantinople le 27 novembre 2013, la célébration liturgique de sa mémoire étant fixée au 2 décembre du calendrier liturgique, le jour de son trépas. Cet évènement, seulement vint-deux ans après son décès, renforce la renommée de Porphyre, ainsi que la valeur probante de son témoignage pour les croyants, y compris aux fins plus subtiles du parcours argumentatif employé dans notre réflexion. Deuxièmement, les développements politiques en Grèce ces dernières années continuent de mettre en 
relief l'écart croissant entre la société civile et l'Église officielle. L'évènement qui représente le mieux cette division est le résultat étonnant du référendum grec du 5 juillet 2015 sur la proposition finale des créanciers. Dans ce scrutin, le «non» a emporté presque $62 \%$ du vote, même si l'establishment politico-économique traditionnel et la hiérarchie de l'Église grecque se sont très ouvertement positionnés en faveur du camp du «oui».

À court et moyen terme, il est fort probable que l'Église orthodoxe grecque continuera de jouir d'une place privilégiée dans l'appareil étatique, mais il est clair que la crise économique a rendu beaucoup plus manifestes les diverses lignes de faille et les aspects les plus problématiques de cette relation Église-État. Notre troisième et dernière observation se rapporte au Saint et grand Concile de l'Église orthodoxe qui s'est déroulé en Grèce, sur l'île de Crète, au mois de juin 2016. Il est vrai que l'ordre du jour du Conseil était très restreint et que les règles de procédure convenues au préalable par la hiérarchie des différentes Églises donnaient peu de place à des discussions de nature plus ouverte. De plus, le refus de certaines Églises importantes d'y participer, l'Église russe en particulier, a affaibli le dessein initial de rassembler toutes les Églises orthodoxes et de démontrer d'une manière pratique l'unité des Orthodoxes à l'échelle mondiale. Cela étant dit, parmi les textes conciliaires résultant de cet exercice se trouve le message final du Saint et grand Concile. Ce texte de portée générale aborde une gamme de sujets, y compris la relation de l'Église avec l'autorité laïque. Plus particulièrement, le message énonce à son paragraphe 10 que:

L'Église orthodoxe n'interfère pas dans le politique. Sa parole reste discrète et prophétique et favorise une intervention humaine appropriée. Les droits de l'Homme sont maintenant au centre de la politique en réponse aux crises politiques et sociales et visent à protéger les citoyens contre l'arbitraire de l'État. Notre Église ajoute également les obligations et les responsabilités des citoyens et la nécessité pour ces derniers d'user de leur autocritique afin d'améliorer sensiblement la société. (Saint et grand Concile de l'Église orthodoxe, 2016, nous soulignons)

Cette déclaration semble viser une relation plus évoluée de l'Église orthodoxe envers l'État qu'auparavant. Nous ne retrouvons aucune référence à l'expérience historique de l’Église sur la relation Église-État, ce qui en soi est peut-être une innovation bienvenue, vu les divergences du discours historique sur cette question ${ }^{7}$. D'ailleurs, la portée exacte de la pres-

7. Nous avons développé ailleurs la thèse concernant la prédominance injustifiée du discours de la symphonie byzantine (Giocas 2014). 
cription excessivement vulgarisée de non-interférence "dans le politique » est loin d'être claire. Nous imaginons pourtant que le choix d'un langage vague et inexact était peut-être nécessaire pour faire adopter le texte par les différentes délégations d'évêques participantes. Ce manque de précision laisse la place à divers choix interprétatifs de ce qui pourrait être souhaitable dans un contexte particulier, et n'exclut pas, ou du moins pas clairement, la possibilité qu'une Église soit intégrée dans l'État d'une manière indésirable ou sectaire.

D’autre part, la référence simpliste au «politique» nous parait grossièrement déplacée face à l'existence de théorisations plus élaborées visant à valider une participation chrétienne progressiste à la politique moderne, comme celle évoquée et défendue par Papanikolaou (2016), dont nous discutons davantage ailleurs ${ }^{8}$. Alors, les questions abordées dans cette courte réflexion demeurent entièrement pertinentes et non résolues. Et même si aucun consensus clair ne se dégage pour le moment, nous espérons que l'idée qu'un processus d' "autocritique ", tel que souligné dans l'extrait du message conciliaire cité ci-dessus, nous amènera des résultats plus concrets dans un avenir proche. Bien sûr, cela nécessitera un approfondissement de plusieurs des questions soulevées d'une manière forcément succincte à travers le corps de notre courte réflexion.

\section{Références}

Athanase D'Alexandrie (1994), Vie d'Antoine / trad. par G. J. M. Bartelink, Paris, Cerf (Sources chrétiennes 400).

BeHr-Sigel, É. (1989), Le Lieu du cour. Initiation à la spiritualité de l'Église orthodoxe, Paris, Cerf.

Deseille, P. (2003), La Spiritualité orthodoxe et la philocalie, Paris, Albin Michel.

Festugière, A.-J. (1961), Les moines d'Orient, vol. 1, Paris, Cerf.

Florovsky, G. (1957), "Empire and Desert. Antinomies of Christian History ", Greek Orthodox Theological Review, 3, p. 133-159.

Fox News KVOA TV 4 (2006), "Mystery Monastery (9 février), disponible sur: <http://www.youtube.com/watch ? v=wJMvc5GERvk>.

Getcha, J. (2009), Le Typikon décrypté. Manuel de liturgie byzantine, Paris, Cerf.

8. Voir notre essai critique qui porte en partie sur la thèse de Papanikolaou (Giocas 2015). 
Giocas, A. (2014), «The Byzantine Legacy of Religious and Legal Pluralism. A Contemporary Reassessment of Byzantine Church-State Relations ", Oxford Journal of Law and Religion, 3, p. 462-483.

(2015), «Christian Practice as the Foundation for Modern Political Theology. Recension de A. Papanikolaou, The Mystical as Political. Democracy and Non-Radical Orthodoxy et de K. De Wolf, Dissident for Life. Alexander Ogorodnikov and the Struggle for Religious Freedom in Russia", Journal of Law and Religion, 30, p. 161-169.

Hustak, A. (1992), "Controversy Rages over Dead Monk's Property. Man Shot Himself after Greek Orthodox Diocese Refused to Ordain Him », The Montreal Gazette, 4 avril, A3.

IoAnnidis, K. (2005), Géron Porphyrios. Témoignages et expériences, Athènes, Saint Couvent de la Transfiguration du Sauveur.

Papanikolaou, A. (2012), The Mystical as Political. Democracy and NonRadical Orthodoxy, Notre Dame, University of Notre Dame Press.

Papastathis, C. K. (2001), «Religious Self-Administration in the Hellenic Republic », dans G. RobBers, dir., Church Autonomy. A Comparative Survey, Francfort-sur-le-Main, Peter Lang, c. 20.

Porphyrios (2005), Wounded by Love. The Life and the Wisdom of Elder Porphyrios / trad. par J. Raffan, Evia, Denise Harvey.

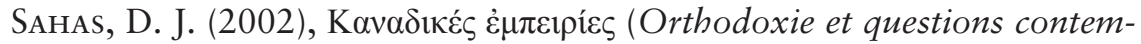
poraines, vol. III: Expériences canadiennes), Waterloo, Université de Waterloo.

Saint et GRand Concile De L'Église orthodoxe (2016), «Message du saint et grand Concile de l'Église orthodoxe au peuple orthodoxe et à toute personne de bonne volonté ", disponible sur: <https://www. holycouncil.org/-/message?_101_INSTANCE_VA0WE2pZ4Y0I_ languageId=fr_FR $>$.

Schmemann, A. (1997), The Historical Road of Eastern Orthodoxy / trad. par L. W. Kesich, Crestwood, St Vladimir's Seminary Press.

St. Anthony's Greek Orthodox Monastery, "Our Affiliated Monasteries in North America ", disponible sur <http://www.stanthonysmonastery.org/map.php>.

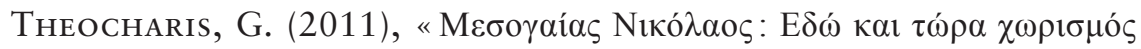

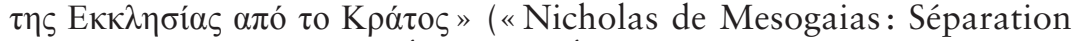
ici et maintenant entre l'Église et l'État»), 14 juillet, disponible sur <http://www.agioritikovima.gr/2011-07-14-22-28-56/2353-mesogaiasnikolaos>. 


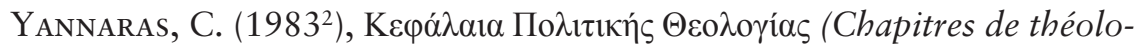

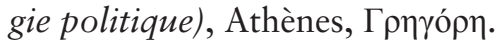

\section{Résumé}

Pour mettre en œuvre un modèle ecclésiologique qui corresponde à l'enseignement de l'ancien Porphyre, la relation intime entre histoire et théologie doit être plus profondément appréciée. Dans cet esprit, l'article expose les grandes lignes d'une réflexion plus large sur le parcours historique du monachisme orthodoxe, se référant à la conception traditionnelle du développement du monachisme au $\mathrm{IV}^{\mathrm{e}}$ siècle, ainsi qu'à certaines expressions plus contemporaines du monachisme. Dans tous les cas, le monachisme agit comme une sorte de contrepoids à la présence continue de forces sécularisantes qui agissent sur la réalité ecclésiale de l’Église et sa mission.

\section{Abstract}

In seeking to implement an ecclesiological model in keeping with the wisdom of Elder Porphyrios, the intimate relationship between history and theology must be more deeply appreciated. In this vein, the article outlines the possibility of a broader reflection on the historical journey of Orthodox monasticism on the basis of the traditional understanding of the development of monasticism in the $4^{\text {th }}$ century, as well as with respect to specific contemporary expressions of monasticism. In all instances, monasticism can be seen as compensating for the ongoing presence of significant secularizing challenges to the ecclesial reality of the Church and its mission. 